\title{
USO DO SENSORIAMENTO REMOTO E DE VEÍCULOS AÉREOS NÃO TRIPULADOS NO MONITORAMENTO DO PROCESSO EROSIVO NO LITORAL DO EXTREMO SUL DO RIO GRANDE DO SUL, BRASIL
}

\author{
Miguel da Guia Albuquerque ${ }^{(a)}$, Deivid Leal Alves $^{(b)}$ Jean Marcel de Almeida Espinoza $^{(\mathrm{c})}$
}

(a) Pós Graduação em Geografia, Universidade Federal do Rio Grande, migueldaguia @ gmail.com

(b) Departamento de Geoprocessamento, Instituto Federal do Rio Grande do Sul, dclealalves@gmail.com,

(c) Departamento de Geoprocessamento, Instituto Federal do Rio Grande do Sul, jean.espinoza@ riogrande.ifrs.edu.br

\section{Eixo: DINÂMICA E GESTÃO DE ZONAS COSTEIRAS}

\begin{abstract}
Resumo
O presente estudo tem como objetivo caracterizar o processo erosivo ocorrido no litoral do extremo sul do Brasil, a partir de sensores remotos e uso de veículos aéreos não tripulados (drone). O estudo se concentrou nos efeitos da passagem do ciclone de 27 de outubro de 2016 na região da praia do Hermenegildo, buscando caracterizar o comportamento da linha de costa e as taxas de erosão antes e após a passagem do evento meteorológico. Um comparativo entre uma imagem de satélite obtida em julho de 2016 e um levantamento realizado com drone em novembro de 2016 mostrou que a linha de costa apresentou uma retração de $6,38 \mathrm{~m} /$ dia. O resultado destaca a importância das oscilações climáticas dominantes e faixas de tempestade na retração da linha de costa durante a passagem de eventos extremos, sendo esse tipo de estudo uma ferramenta básica de interesse geral da gestão costeira.
\end{abstract}

Palavras chave: sensores remotos, geoprocessamento, geotecnologias

\section{Introdução}

As zonas costeiras são regiões submetidas a um processo intenso de ocupação, que precisa ser analisado e monitorado para subsidiar os planos de Gestão Pública. Embora grande parte da população mundial esteja alocada próxima às zonas costeiras, existem poucos estudos que enfoquem os impactos ambientais e desastres naturais relacionados à ação de eventos extremos. Para o litoral sul do Brasil, alterações nos ciclos climáticos têm sido observadas desde a década de 1990 (MACHADO et al., 2010). Estudos relacionados aos impactos dos eventos extremos na costa do Rio Grande do Sul têm sido realizados com intuito de descrever a vulnerabilidade e riscos existentes. Dessa forma, a ação dos gestores municipais devem ser direcionadas para o mapeamento da frequência e modo de ocorrência desses eventos extremos, bem como a implementação de medidas mitigatórias e preventivas que possam minimizar os impactos causados por esses fenômenos (ALBUQUERQUE et al., 2015). A partir do exposto, o presente estudo visa caracterizar o comportamento das praias do litoral do extremo sul do Rio Grande do Sul após a passagem de tempestades. 


\section{1 Área de Estudo}

O estudo se concentrou na praia do Hermenegildo, situada no extremo sul do Brasil, a $16 \mathrm{~km}$ ao norte da barra do Chuí (Figura 1). A localidade possui uma costa dominada por ondas, com ventos predominantes do quadrante NE e uma maré do tipo semidiurna, com altura média de $0,30 \mathrm{~m}$. Os períodos de ressaca têm sido registrados nos meses de abril e maio, e em geral a maré meteorológica pode alcançar 1,20 m (CALLIARI et al., 1996). Em termos morfodinâmicos a praia do Hermenegildo é classificada no estágio morfodinâmico intermediário com tendência a dissipativo, segundo a classificação de Wright \& Short (1984). A localidade é caracterizada por um intenso processo erosivo que, nos últimos anos, tem se agravado devido ao conflito entre a atuação dos eventos extremos e a atividade antrópica.

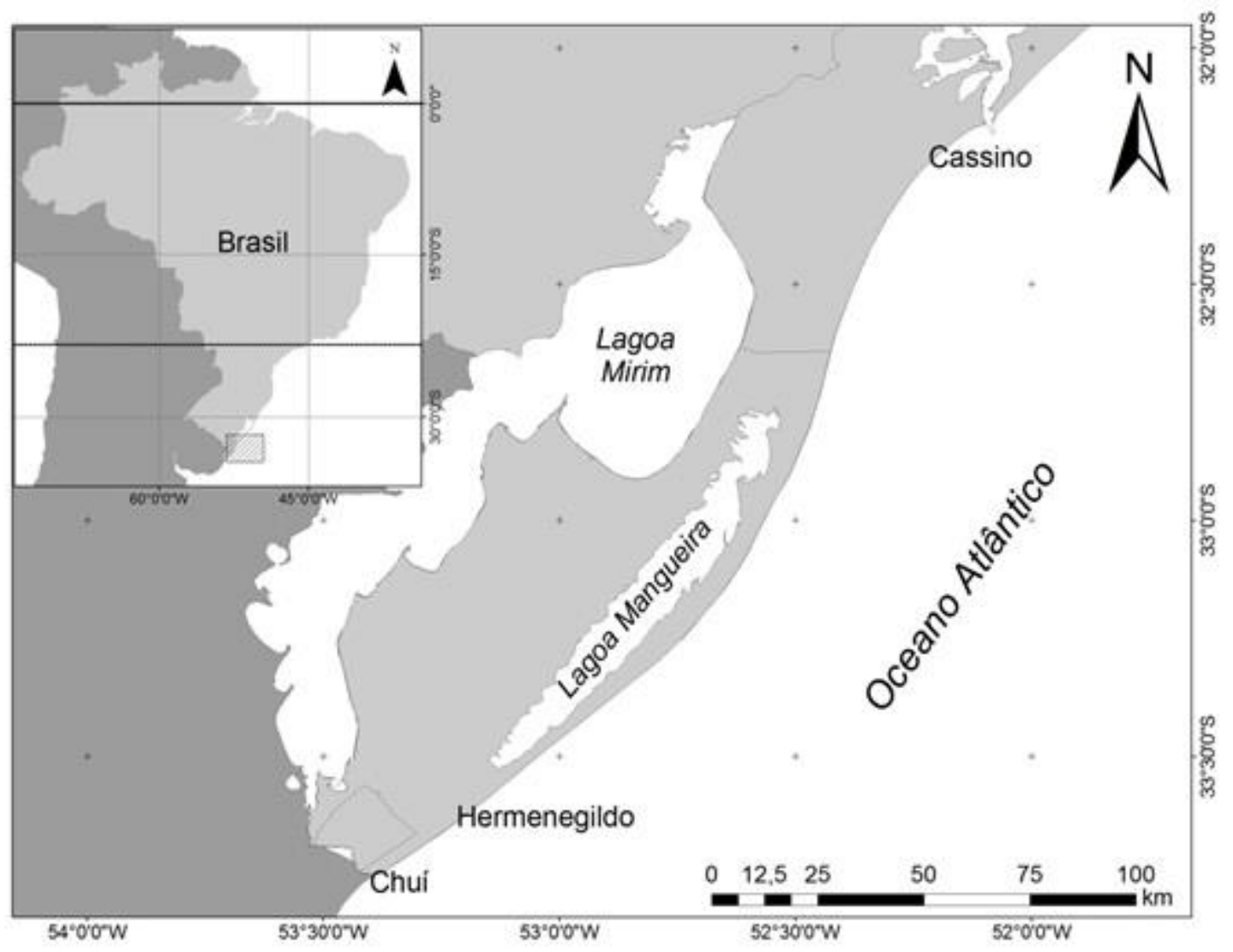

Figura 1- Mapa de localização da área de estudo.

\section{Materiais e Métodos}

Para o monitoramento da linha de costa da praia do Hermenegildo foram utilizados dados do sensor Geoeye e de um Veículo Aéreo Não Tripulado (VANT/ DRONE) equipado com uma câmera Nikon 


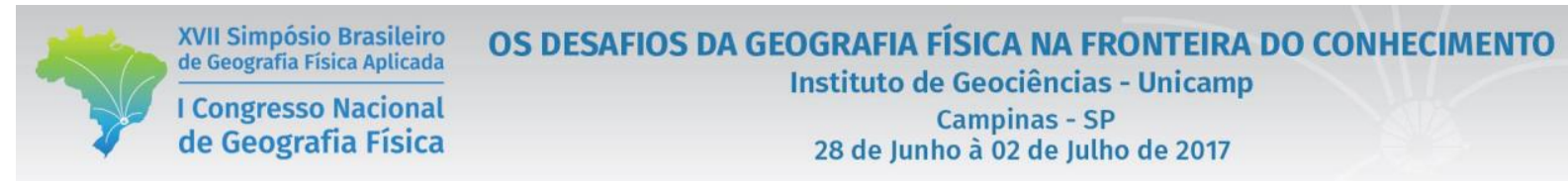

Coolpix A, a qual possui um CCD de 24 megapixels. A imagem do sensor Geoeye foi coletada no período de julho de 2016, com resolução espacial de 0,5 m. A campanha com o Drone foi realizada no dia 30 de novembro de 2016 e registrou, aproximadamente, 446 milhões de pontos, com resolução espacial de 0,21 m. O monitoramento exigiu dois voos, que cobriram quase $2,5 \mathrm{Km}^{2}$ com sobreposição de $60 \%$ e altura fixa a 40 m. Foi utilizada uma sobreposição elevada, de acordo com Haala et al. (2013), tendo em vista a possibilidade de uma intersecção multi-raio, o que ajudou na obtenção de uma melhor precisão e densidade da nuvem de pontos.

A orientação das imagens foi feita no software Agisoft Photoscan ${ }^{\circledR}$, de forma que foi possível extrair a nuvem de pontos, construir um modelo digital de superfície e produzir mosaicos de ortoimagens. O processo de triangulação aérea, a calibração da câmera e a geração do modelo foram automatizados, sendo que todo o processo usou um GPS-RTK para obter uma melhor precisão posicional.

O cálculo das taxas de recuo da linha de costa foram realizados a partir do método do polígono de mudança (SMITH \& CROMLEY, 2012; ALBUQUERQUE et al., 2013). A metodologia consiste em extrair áreas erodidas e/ou acrescidas, a partir de duas linhas de costa distintas previamente vetorizadas, através da geração e subtração de polígonos (Figura 2). Na álgebra de polígonos toda região de variação da linha de costa é quantificada, de modo que o cálculo do deslocamento é feito pela divisão entre a área de praia (A) e o comprimento do segmento costeiro (L).

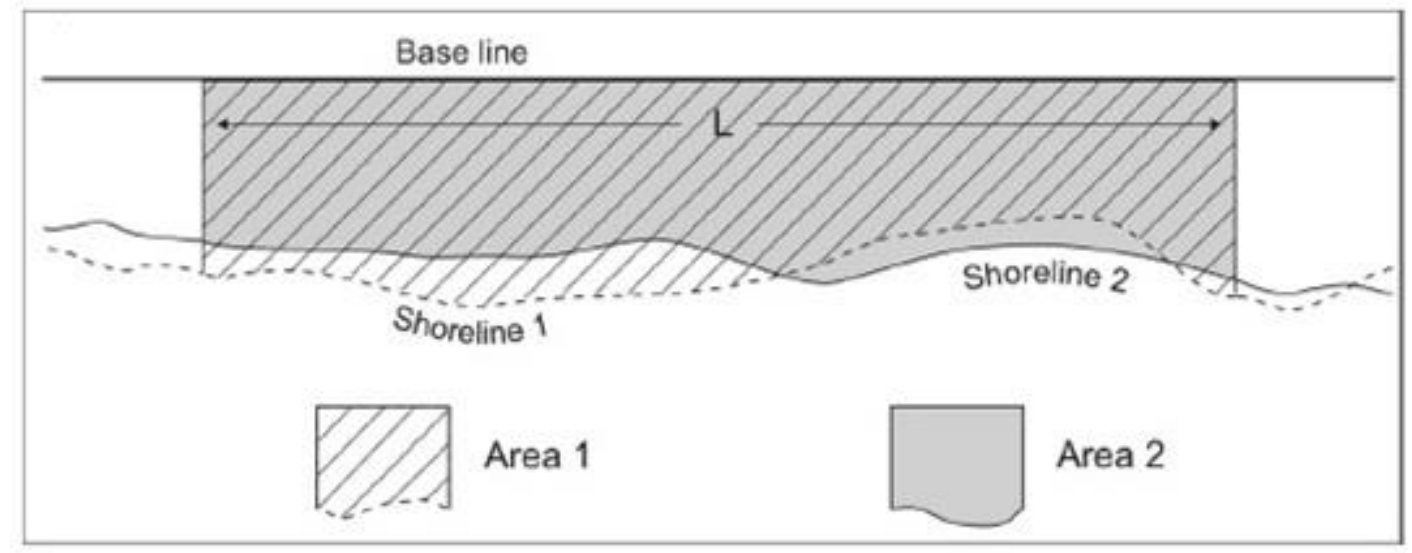

Mean shoreline displacement $=($ Area $2-$ Area 1$) / \mathrm{L}$

Figura 2: Caracterização do método do polígono de mudança. Fonte: Extraído de Anfuso et al. (2016).

\section{Resultados e Discussões}

Para o evento meteorológico ocorrido em 27 de outubro de 2016, a comparação dos dados de posição da 


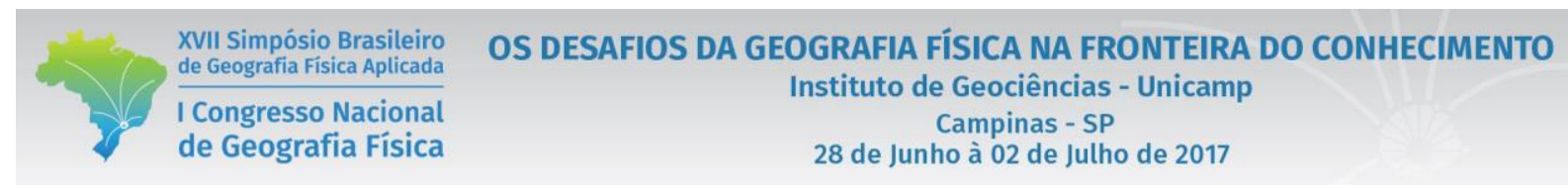

linha de costa obtidos pela imagem do sensor Geoeye de julho de 2016 e do Drone (obtida em novembro de 2016) mostrou uma taxa média de recuo de 6,75 m em relação aos quatro meses. Quando comparado às retrações da linha de costa obtidas por Albuquerque (2013), para uma série temporal de dados iniciada em 1947, este evento foi considerado o mais destrutivo dos últimos 69 anos. Durante a passagem do ciclone (Figura 3) o vento apresentou uma velocidade de $80 \mathrm{Km} / \mathrm{h}$ com direção S-SW. Este evento causou impactos físicos extensos (erosão de praias e dunas) e socioeconômicos (inundações e danos às infraestruturas) em toda a praia do Hermenegildo. A região norte do balneário foi o mais atingido, com uma alta retração do campo de dunas. Na porção sul, cerca de 40 residências foram danificadas.

\section{Press_00Z270CT2016}

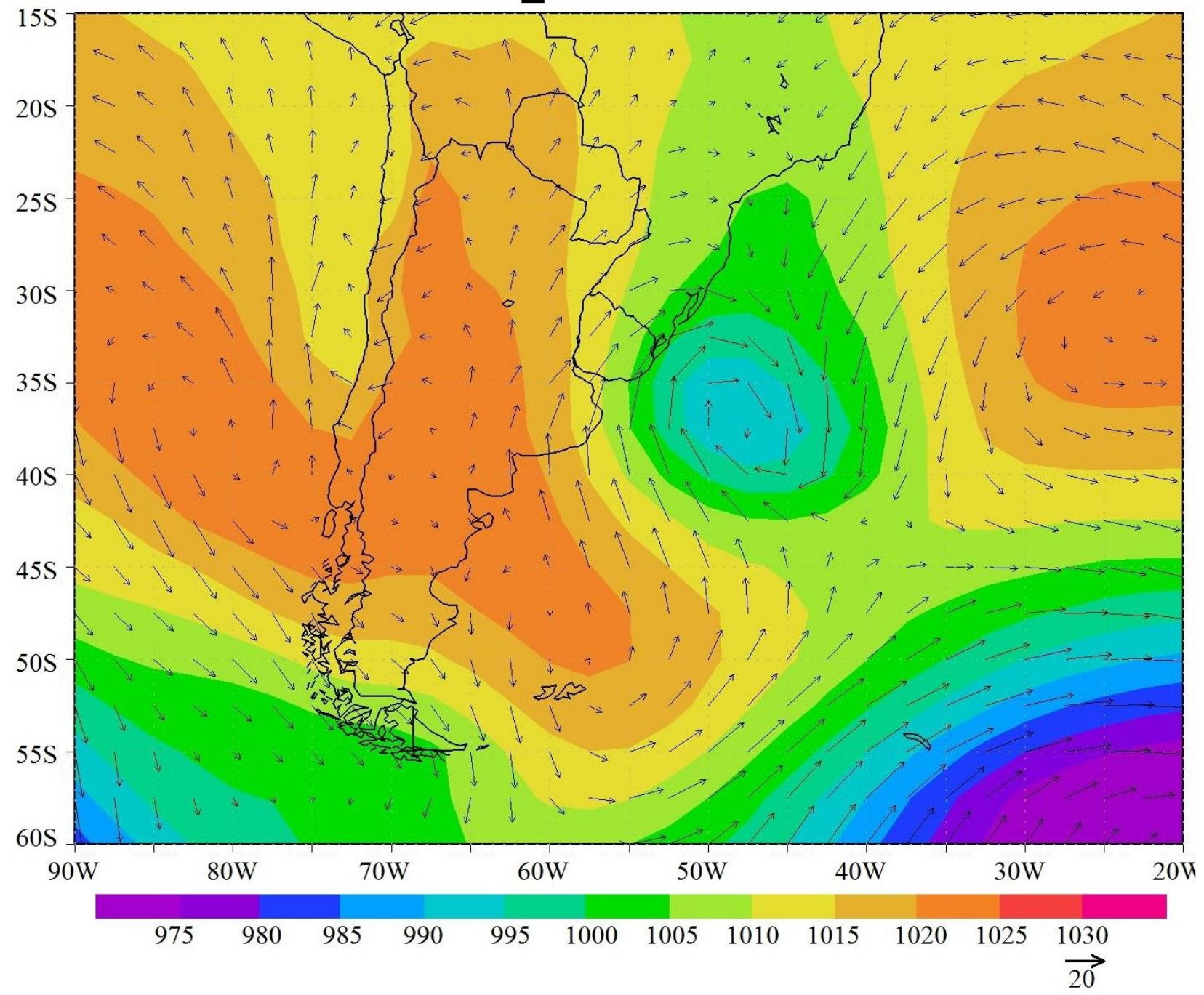

Figura 3: Situação sinótica do dia 27 de outubro de 2016, com formação de ciclone próximo à costa.

O balanço final aproximado do deslocamento da linha de costa foi de 6,38 $\mathrm{m} /$ dia (Figura 4), para o ciclone 


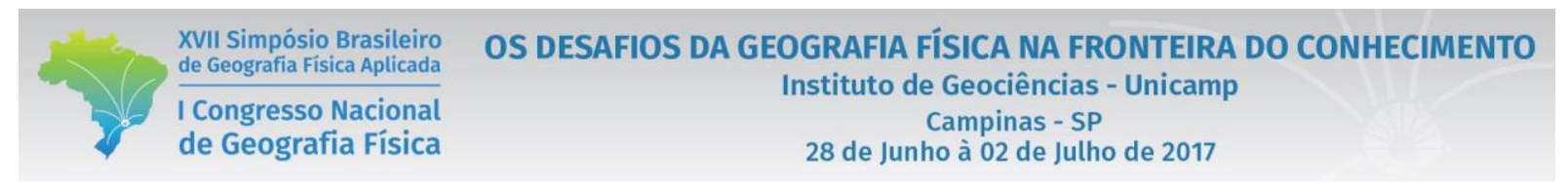

ocorrido entre 26 e 27 de outubro de 2016. Esse resultado foi obtido partir da retração calculada entre julho novembro de $2016(6,75 \mathrm{~m})$ subtraída o recuo médio do litoral $(0,365 \mathrm{~m})$ calculado por Albuquerque (2013), a partir de levantamentos semanais ocorridos entre julho e novembro de 2012, com uso de um GPS-RTK.

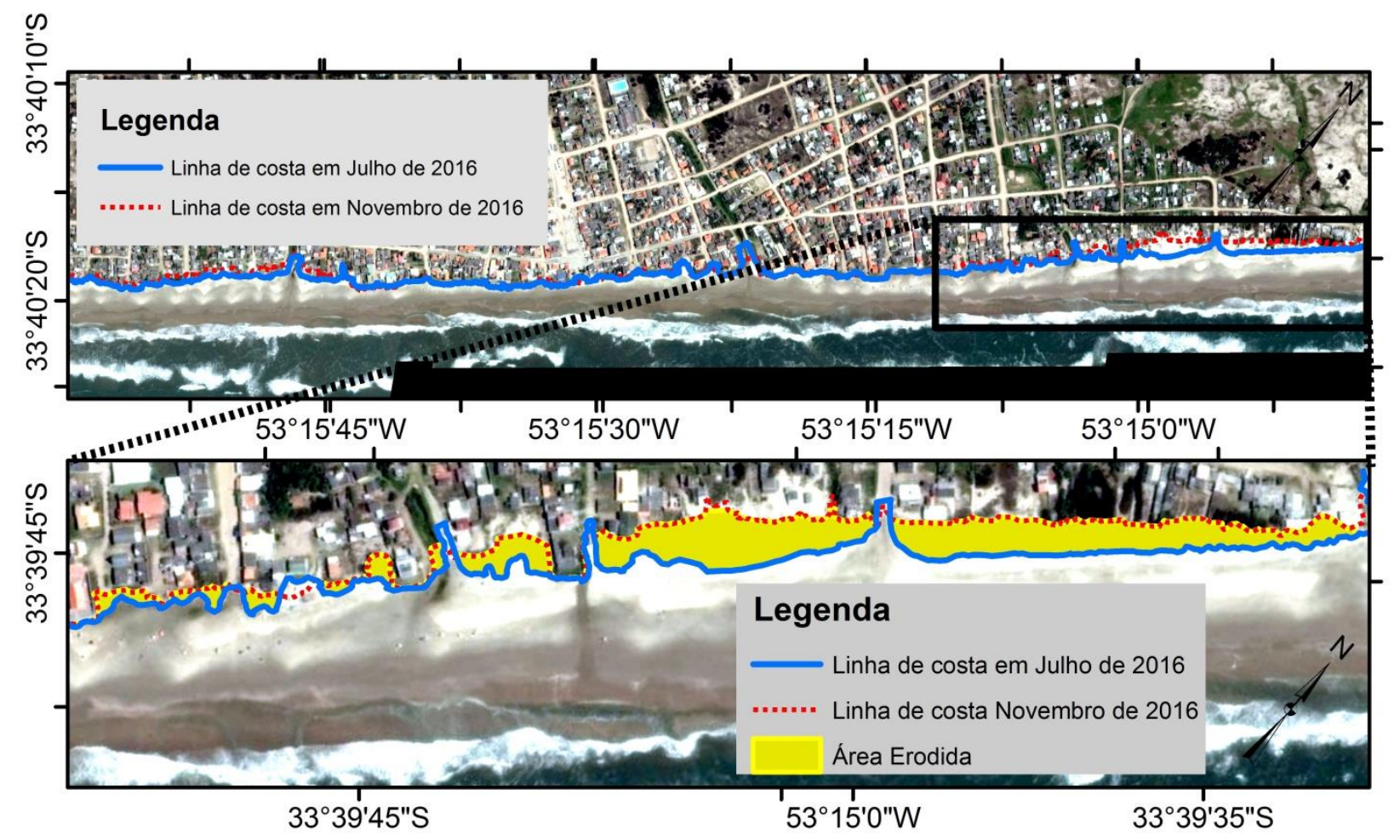

Figura 4: Deslocamento da linha de costa entre julho e novembro de 2016.

\section{Conclusões}

Os recentes acontecimentos têm aumentado a preocupação dos moradores e gestores costeiros sobre os efeitos devastadores das ondas de tempestade, com um aumento do nível do mar muito além do normal. Quando comparado o evento ocorrido em outubro de 2016 com os principais eventos de tempestades incidentes no extremo sul do Brasil, observou-se que estes apresentavam ventos predominantes de S-SW no período do evento. Em geral os ciclones contribuem para um aumento do nível do mar local, o que também contribui para aumentar as taxas de retração do litoral.

O nosso entendimento da resposta dos ciclones é limitada pela qualidade e adequação dos conjuntos de dados disponíveis, particularmente para medições qualitativas ao longo de uma sequência completa de recuperação da praia. O conhecimento dos processos de mudança do litoral aumenta a capacidade dos gestores costeiros para gerir os riscos, especialmente a erosão costeira associada a surtos de tempestades. 


\section{REFERÊNCIAS}

ALBUQUERQUE, M.G.; LEAL ALVES, D.C.; MACHADO, A.; ESPINOZA, J.; CALLIARI, L.; GANDRA, T. Gestão costeira e riscos associados a tempestades: um panorama dos impactos costeiros causados pelos eventos extremos no Rio Grande do Sul. In: DE PAULA, D; DIAS, J.A. (org.), Ressacas do Mar/ Tempestades e Gestão Costeira. Fortaleza: Editora Premius, 2015. pp. 415-444.

AlbuQUerQUe, M.G.; ESPINOZA, J.; TEIXEIRA, P.; DE OlIVEIRA, A.; CORREA, I.; CALliARI, L. Erosion or coastal variability: an evaluation of the DSAS and change polygon methods for the determination of erosive process on sandy beaches - Journal of Coastal Research, SI(65): 1710-1714, 2013.

ALBUQUERQUE, M. G. Análise espaço-temporal das causas da variabilidade da linha de costa e erosão na praia do Hermenegildo, RS. 2013. 127 f. Tese (Doutorado em Geociências) - Programa de Pós-Graduação em Geociências, Universidade Federal do Rio Grande do Sul.

ANFUSO, G.; BOWMAN, D.; DANESE, C.; PRANZINI, E. - Transect based analysis versus area based analysis to quantify shoreline displacement: spatial resolutions issues - Environmental Monitoring Assessment, 188: 568, 2016.

CALLIARI, L.J.; KLEIN, A.H.F.; BARROS, F.C.R. - Beach differentiation along the Rio Grande do Sul coastline (southern Brazil). Rev. Chil. Hist. Nat., 69: 485-493, 1996.

HAALA, N.; CRAMER, N.; ROTHERMEL, M. - Quality of 3D point clouds from highly overlapping UAV imagery. International Archives of the Photogrammetry, Remote Sensing and Spatial Information Science, 40(1W2): 183-188, 2013.

MACHADO, A.A.; CALLIARI, L.J.; MELO, E.; KLEIN, A.H.F. - Historical assessment of extreme coastal sea state conditions in southern Brazil and their relation to erosive episodes. Pan-American Journal of Aquatic Sciences, 5(2): 105-114, 2010.

SMITH, M.J. \& CROMLEY, R.G. - Mensure historical coastal change using GIS and the change polygon approach. Transactions in GIS, 16(1): 3-15, 2012.

WRIGHT, L.D.; SHORT, A.D. - Morphodynamic variability of surf zones and beaches: a synthesis. Marine Geology, 56: 93-118, 1984. 